\title{
Necessity of prophylactic splenic hilum lymph node clearance for middle and upper third gastric cancer: a network meta- analysis
}

Gaozan Zheng ${ }^{1 \dagger}$, Jinqiang Liu' ${ }^{1,2+}$, Yinghao Guo ${ }^{1,3+}$, Fei Wang ${ }^{1,4}$, Shushang Liu', Guanghui Xu', Man Guo ${ }^{1}$, Xiao Lian', Hongwei Zhang ${ }^{1 *}$ (D) and Fan Feng ${ }^{1 *}$

\begin{abstract}
Background: It remains controversial whether prophylactic No.10 lymph node clearance is necessary for gastric cancer. Thus, the present study aims to investigate the impact of prophylactic No.10 lymph node clearance on the perioperative complications and prognosis of upper and middle third gastric cancer.

Methods: A network meta-analysis to identify both direct and indirect evidence with respect to the comparison of gastrectomy alone (G-A), gastrectomy combination with splenectomy $(G+S)$ and gastrectomy combination with spleen-preserving splenic hilar dissection (G + SPSHD) was conducted. We searched Medline, Embase, and the Cochrane Central Register of Controlled Trials (CENTRAL) for studies published before September 2018. Perioperative complications and overall survival were analyzed. Hazard ratios (HR) were extracted from the publications on the basis of reported values or were extracted from survival curves by established methods.

Results: Ten retrospective studies involving 2565 patients were included. In the direct comparison analyses, G-A showed comparable 5-year overall survival rate (HR: 1.1, 95\%Cl: 0.97-1.3) but lower total complication rate (OR: 0.37, 95\%Cl: 0.170.77) compared with $G+S$. Similarly, the 5-year overall survival rate between $G+$ SPSHD and $G+S$ was comparable (HR: 1.1, 95\%Cl: 0.92-1.4), while the total complication rate of G + SPSHD was lower than that of G + S (OR: 0.50, 95\%Cl: 0.280.88). In the indirect comparison analyses, both the 5-year overall survival rate (HR: 1.0, 95\%Cl: 0.78-1.3) and total complication rate (OR: 0.75, 95\%Cl: 0.29-1.9) were comparable between G-A and G + SPSHD.
\end{abstract}

Conclusions: Prophylactic No.10 lymph node clearance was not recommended for treatment of upper and middle third gastric cancer.

Keywords: Gastric cancer, Splenic hilar, Lymph node, Splenectomy, Spleen-preserving

\section{Background}

Gastric cancer remains the second most common cause of cancer-related death worldwide despite a decline in incidence [1]. Surgery is the main treatment for patients with gastric cancer. Even when surgery is combined with perioperative chemotherapy and/or radiation, outcomes

\footnotetext{
* Correspondence: zhanghwfmmu@126.com; surgeonfengfan@163.com †'Gaozan Zheng, Jinqiang Liu and Yinghao Guo contributed equally to this work.

${ }^{1}$ Department of Digestive Surgery, Xijing Hospital of Digestive Diseases, the Fourth Military Medical University, 127 West Changle Road, Xi'an 710032, Shaanxi, China

Full list of author information is available at the end of the article
}

remain poor. Therefore, various perioperative treatment strategies have been investigated to improve the surgical outcomes. Previous studies reported that D2 lymphadenectomy could achieve curability and prolong survival of gastric cancer [2]. Furthermore, it is generally accepted that gastrectomy with more lymph nodes resected could decrease recurrence rates and improve survival of gastric cancer patients [3]. While extended lymphadenectomy is associated with improved locoregional disease control, decreased recurrence rates, and improved DSS, it seems inappropriate to claim D2 lymphadenectomy could "achieve curability."

(c) The Author(s). 2020 Open Access This article is distributed under the terms of the Creative Commons Attribution 4.0 International License (http://creativecommons.org/licenses/by/4.0/), which permits unrestricted use, distribution, and reproduction in any medium, provided you give appropriate credit to the original author(s) and the source, provide a link to the Creative Commons license, and indicate if changes were made. The Creative Commons Public Domain Dedication waiver (http://creativecommons.org/publicdomain/zero/1.0/) applies to the data made available in this article, unless otherwise stated. 
The frequency of lymph node metastasis (LNM) to the splenic hilum and splenic artery has been reported as 10$20 \%$ in upper and middle third gastric cancer $[4,5]$, and the 5-year overall survival rate of patents with splenic hilar lymph node metastasis (SHLNM) was significantly lower than those without SHLNM [6-9]. Therefore, it is necessary to dissect No.10 lymph node by splenectomy or spleen-preserving splenic hilar dissection (SPSHD) for patients with evident macroscopic enlarged lymph node at the splenic hilar. However, for patients without enlarged splenic hilar lymph node, the value of prophylactic No.10 clearance for the prognosis of gastric cancer patients remains controversial. A series of studies emphasized the necessity of lymph node dissection at the splenic hilum to remove the potentially affected lymph nodes [10-13]. However, some investigators reported that No.10 lymphadenectomy was not associated with improved oncologic outcomes but was only associated with increased rates of postoperative complications [14-16].

Thus, the aim of this study was to evaluate the impact of prophylactic No.10 lymph node clearance on the perioperative complications and prognosis of middle and upper third gastric cancer.

\section{Methods}

\section{Search strategy and selection criteria}

For this network meta-analysis, Two investigators (Z.G and L.J) searched PubMed, the Cochrane Central Register of Controlled Trials (CENTRAL) and Embase for both retrospective study and randomized controlled trials (RCTs) published from the date of database inception to September 2018, of which the search strings were based on MeSH terms: "Stomach Neoplasms [mesh] OR Neoplasm, Stomach [title] OR Stomach Neoplasm [title] OR Neoplasms, Stomach [title] OR Gastric Neoplasms [title] OR Gastric Neoplasm [title] OR Neoplasm, Gastric [title] OR Neoplasms, Gastric [title] OR Cancer of Stomach [title] OR Stomach Cancers [title] OR Gastric Cancer [title] OR Cancer, Gastric [title] OR Cancers, Gastric [title] OR Gastric Cancers [title] OR Stomach Cancer [title] OR Cancer, Stomach [title] OR Cancers, Stomach [title] OR Cancer of the Stomach [title] OR Gastric Cancer, Familial Diffuse [title] OR Gastrectomies [title] OR Gastrectomy [title] OR gastric resection [title]) AND (Splenectomy [mesh] OR Splenectomies [title] OR spleen dissection [title] OR spleen-preserving [title] OR splenic preservation [title] OR Spleen preservation [title] OR preserving spleen [title] OR Spleen conserving [title] OR reserving Spleen [title] OR spleen preserving [title] OR Spleen-conserving [title] OR Splenic hilar [title] OR splenichilus [title] OR splenic hilum [title] OR hilum of spleen [title] OR No.10[title] OR No. 10[title]". Additionally, we reviewed the reference lists of all the meta-analyses.
Studies with the following situations were considered excluded: (1) Studies with other kinds of gastric tumors, such as lymphoma, other organ tumors or multiple gastric tumors; (2) Studies with splenectomy induced by iatrogenic injury; (3) Studies with splenectomy also underwent distal pancreatectomy; (4) Studies with splenectomy or spleen-preserving splenic hilar dissection induced by enlarged nodes at splenic hilar were excluded; (5) Studies with distal gastric cancer (barely metastasize to splenic hilar lymph node). (6) Studies did not distinguish the $\mathrm{G}+\mathrm{S}$ and $\mathrm{G}+\mathrm{SPSHD}$.

\section{Data extraction and quality assessment}

After removal of duplicates, two investigators (Z.G and G.Y) independently screened all titles and abstracts for eligibility. Any discrepancies were resolved by consensus and arbitration by a panel of other investigators (W.F and L.S) within the review team. Only studies published in full text were included. Experimental studies in animal models, single case reports, technical reports, reviews, abstracts, editorials and studies in languages other than English were excluded after review. If a trial was covered in more than one reports we used a hierarchy of data sources.

Two investigators (Z.G and X.G) independently reviewed the main reports and supplementary materials, extracted the relevant information from the included trials with a predefined data extraction sheet. Extracted data included study characteristics, baseline patient characteristics, intervention details and outcome measures. Risk of bias assessment was conducted by 2 authors in duplicate (G.M and L.X) using the NewcastleOttawa Scale (NOS) system. The maximum possible score is 9 points, and NOS scores greater than six are considered indicative of high-quality studies [17].

\section{Statistical analysis}

The network meta-analysis (NMA) which considers direct and indirect evidence on the benefits and harms among multiple treatments simultaneously, done in line with the items of the Preferred Reporting Items for Systematic Reviews and Meta-Analyses for Network Meta-Analyses (PRISMA-NMA) [18]. Details of statistical approaches applied in this study are provided as follow: The network geometry was used to graphically summarized the relationship of the three treatments (G-A, G+S and G + SPSHD), including both direct and indirect comparison. STATA in this network framework, the information of treatment $\mathrm{G}+$ $\mathrm{S}$ vs. G-A and $\mathrm{G}+\mathrm{S}$ vs. $\mathrm{G}+\mathrm{SPSHD}$ could be extracted directly from the publications. The existing network relationship enables us to construct the indirect comparisons (G-A vs. $G+$ SPSHD) from two trials that have one treatment in common $(G+S)$, and through network meta-analysis to get pooled estimated effects of both direct and indirect treatments. We performed this process using GeMTC (Generate Mixed Treatment Comparisons) package of $\mathrm{R}$ (version 
3.4.3) through calling the code of Winbugs (version 1.4.3, [19]. The Winbugs is a software based on Bayesian Markov chain Monte Carlo which fully preserves randomised treatment comparisons within trials [20-22]. Furthermore, trace plots and the Brooks-Gelman-Rubin [23] statistic were assessed to ensure convergence (Appendix 1).

As measures of the primary treatment, the odds ratio (OR) and the standardized mean difference (SMD) was respectively calculated for dichotomous outcomes and continuous data, both with its $95 \%$ confidence interval (CI). The study endpoint was overall survival rate and the hazard ratio (HR) was assessed for the treatment effects. The HR with 95\% CI were obtained directly from readymade data of the included publications if at all possible. Otherwise, the parameter were estimated through extracting survival information from Kaplan-Meier curves using Engauge Digitizer version 4.1 (free software downloaded from http://sourceforge.net) and converting the survival rate into HRs described by Parmar et al. [24]. Estimates of relative treatment effects was plotted via forest plots and that of rank probabilities was plotted using the rank plot, a rank plot created using the rankogram function from the gemtc $\mathrm{R}$ package visually illustrating probabilities that each treatment is ranked. Above each treatment, the numbers of columns for all treatments in a network relationship is corresponding in a rank plot. The height of the column represents the magnitude of the ranking probability, and the column color from dark to light represents a sort order (1st to last). The higher the ranking, the more recommended the treatment [25]. A common between study heterogeneity parameter I-squared was assumed for all comparisons using 'mtc.anohe' method of the GeMTC $\mathrm{R}$ package. While the inconsistency for a comparison could not be assessed for the reason that the direct and indirect comparison did not co-exist in any branch of the comparison. (version 3.2.2). Heterogeneity was regard as low or high for I-squared values $<25 \%$ or $>75 \%$, respectively [26]. Two-tailed $P$ values of 0.05 were used for statistical significance.

\section{Results}

Treatment strategy network

Overall, 2530 citations were identified by the search and 222 potentially eligible articles were retrieved in full text (Fig. 1). Finally, 10 studies involving 2565 patients which containing any 2 of the 3 surgical procedures (G-A, G +

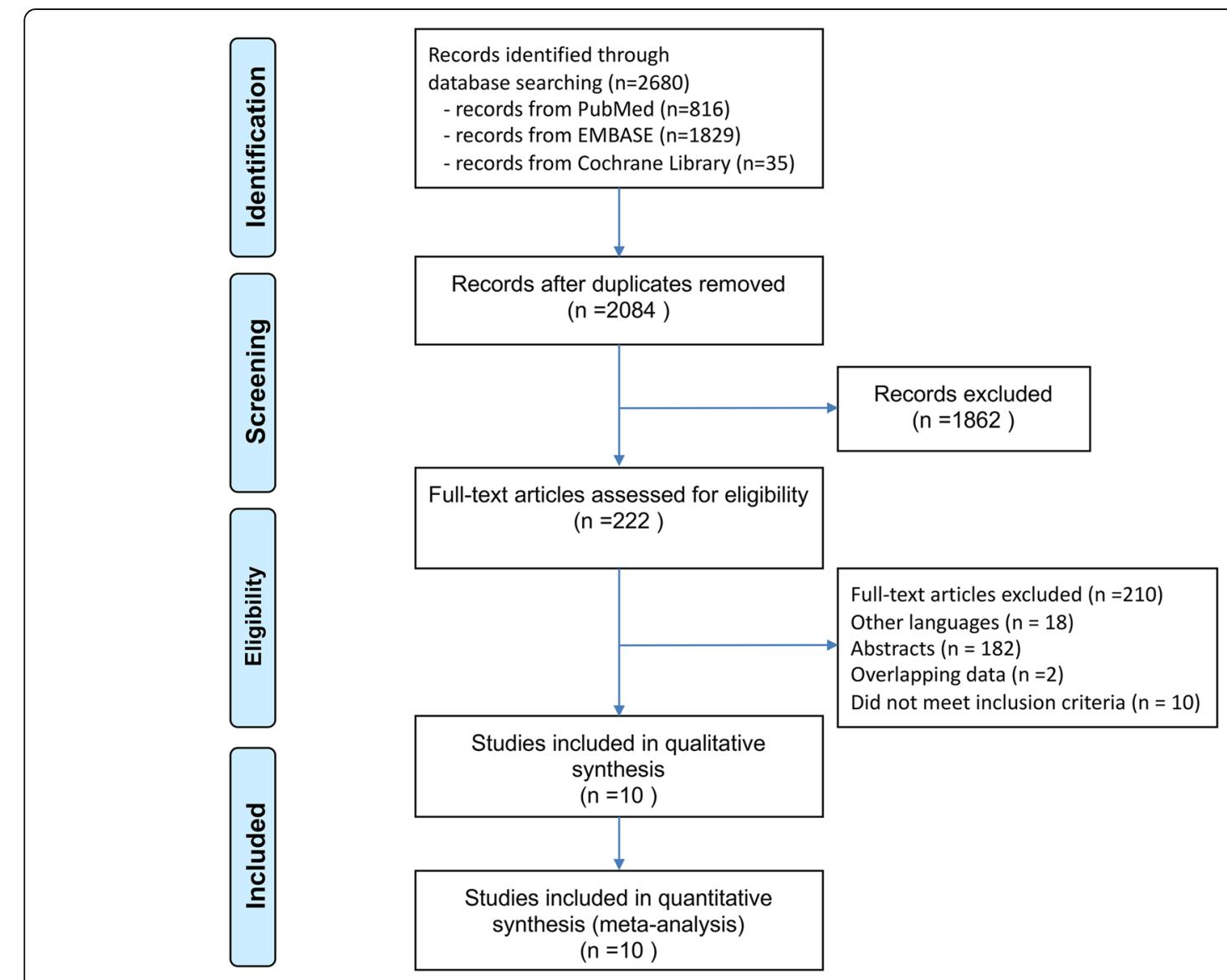

Fig. 1 PRISMA flow chart for the study 
$\mathrm{S}$ and G + SPSHD) were included in the analyses [14, 27-35]. The study characteristics of the publications included in the meta-analysis were shown in Table 1.

The network analysis diagram was shown in Fig. 2. Direct meta-analysis was feasible for the following comparisons: $\mathrm{G}-\mathrm{A}$ versus $\mathrm{G}+\mathrm{S}$ ( 6 trials, $n=1480$ ), and $\mathrm{G}+\mathrm{S}$ versus $\mathrm{G}+$ SPSHD (4 trials, $n=1085$ ). However, G-A with $\mathrm{G}+$ SPSHD could only be compared through indirect meta-analysis.

\section{Risk of bias assessment}

Two independent reviewers (G.M and L.X) evaluated the quality of evidence reported in each study using the Cochrane risk of bias tool. A summary of the risk of bias for each included study is shown in Table 1. All ten articles were scored $\geq 7$, which ensured the high quality of the included articles.

\section{The pooled result of perioperative complications}

The most common complications were anastomotic leakage, pancreas-related complications, bleeding, wound complications, pulmonary and ileus. The results of the network meta-analyses for the perioperative complications was presented in Fig. 3. In the direct comparison analyses, both GA (OR: 0.37, 95\%CI: $0.17-0.77$ ) and $\mathrm{G}+$ SPSHD group (OR: 0.50, 95\%CI: 0.28-0.88) showed lower total complication rate compared with $\mathrm{G}+\mathrm{S}$ group. The indirect comparison showed that the total complication rate of $\mathrm{G}+$ SPSHD group did not differ significant from that of G-A group (OR: 1.3, 95\%CI: 0.52, 3.4).

\section{The network meta-analyses of the 5-year overall survival rate}

As HR in one study [29] was not shown and could not be extracted from survival curves according to the Parmar's method [24], only 9 studies were included for survival analysis. The results from direct and indirect metaanalysis of overall survival rates were shown in forest plot (Fig. 4). In the direct comparison analyses, both G-
A (HR: 1.1, 95\%CI: 0.97-1.3) and G + SPSHD group (HR: 1.1, 95\%CI: 0.92-1.4) showed no significant difference compared with $\mathrm{G}+\mathrm{S}$ group in 5-year overall survival rates. The indirect comparison result showed that the prognosis was also comparable between G-A and G + SPSHD group (HR: 1.0, 95\%CI: 0.78-1.3).

\section{Ranking plots of treatments based on probabilities}

Similar to the above trend, the rank plot showed that the G-A group is the optimal intervention because it has the highest probability of being ranked first, followed by the $G+$ SPSHD group and the last one is the $G+S$ group (Fig. 5).

\section{Discussion}

The spleen is rarely a target of direct invasion by gastric cancers, but sometimes LNM is found in splenic hilus. For that reason, prophylactic removal of the splenic hilar lymph nodes in gastric cancer has been advocated by some investigators. However, it remains inconclusive whether prophylactic clearance of lymph node station No. 10 is associated with overall survival in patients undergoing resection of gastric cancer. Thus, the present study aims to investigate the impact of prophylactic No.10 lymph node clearance on the perioperative complications and prognosis of middle and upper third gastric cancer. This is the first analysis to compare the impact of G-A, $\mathrm{G}+\mathrm{S}$ and $\mathrm{G}+$ SPSHD on the perioperative complications and prognosis of middle and upper third gastric cancer. Our findings indicated that prophylactic No.10 lymph node clearance was not recommended for treatment of upper and middle third gastric cancer.

To date, three prospective randomized controlled trials (RCTs) have evaluated the impact of prophylactic splenectomy on prognosis in patients with gastric cancer. The Csendes trial [36] enrolling 187 patients in a single institution showed that splenectomy has no effect on survival after total gastrectomy ( $42 \%$ vs $36 \%$ ). The Japanese trial [37] enrolling 505 patients in multicenter showed no

Table 1 Characteristics of included studies

\begin{tabular}{|c|c|c|c|c|c|c|}
\hline Author & Year of Publication & Country & Group & No of patients & Tumor Location & Quality score \\
\hline Erturk & 2003 & Turkey & $G-A G+S$ & 2338 & Upper, Middle & 7 \\
\hline $\mathrm{Ji}$ & 2016 & China & $G-A G+S$ & 243,105 & Upper & 8 \\
\hline Lee & 2001 & Korea & $G-A G+S$ & 173,492 & Upper, Middle, Whole & 8 \\
\hline Kodera & 1997 & Japan & $G-A G+S$ & 57,129 & Upper & 7 \\
\hline Ohkura & 2017 & Japan & $G-A G+S$ & 4563 & Upper & 7 \\
\hline Yao & 2011 & China & $G-A G+S$ & 6151 & Upper, Whole & 7 \\
\hline Fang & 2012 & Taiwan & $\mathrm{G}+\mathrm{SPSHD} \mathrm{G}+\mathrm{S}$ & 6847 & Upper & 7 \\
\hline Kwon & 1997 & Korea & $G+S P S H D G+S$ & 232,260 & Upper, Middle, Whole & 8 \\
\hline Oh & 2009 & Korea & $\mathrm{G}+\mathrm{SPSHD} \mathrm{G}+\mathrm{S}$ & 26799 & Upper & 8 \\
\hline Son & 2017 & Korea & $G+S P S H D G+S$ & 6844 & Upper, Middle, Whole & 7 \\
\hline
\end{tabular}




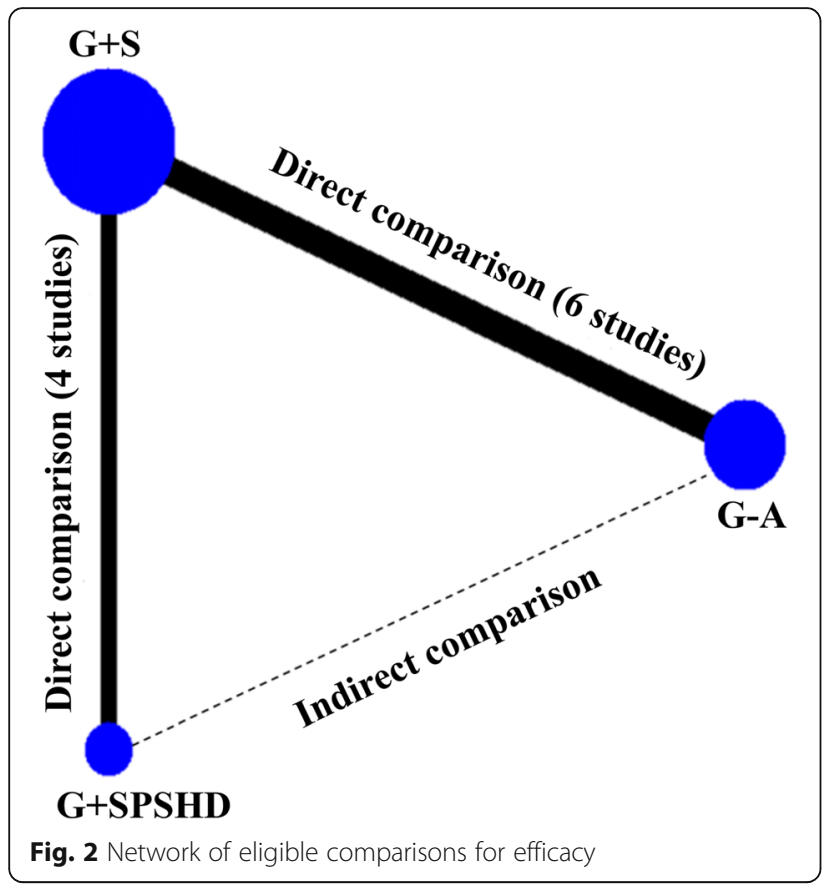

survival difference between $\mathrm{G}+\mathrm{S}$ group and G-A group $(75.1 \%$ vs $76.4 \%)$ in upper third gastric cancer. Another small-scale trial [38] enrolling 79 patients reported by Toge et al. showed a slightly better 5-year survival with splenectomy, but the difference was not statistically significant either. In our present study, splenectomy is not recommended for gastric cancer patients without direct invasion of the splenic hilar lymph nodes because this procedure does not increase the survival rate, but only increase the postoperative complications.
Many studies have assessed the role of prophylactic splenectomy in gastric cancer patients with high risk factors for No.10 lymph node metastasis. Ohno et al. suggested that $\mathrm{G}+\mathrm{S}$ is the optimal procedure in proximal T3 gastric cancer [9]. However, Ito et al. reported that, in patients with pT3-4 tumors, prophylactic splenectomy has no significant survival benefit [39]. Furthermore, studies show that there was no significant difference in recurrence rate and 5-year survival rate at stage III and IV the patients who underwent total gastrectomy with or without splenectomy $[8,14,29,40]$. With respect to tumor location, Ohkura et al. found that prophylactic splenectomy has no significant prognostic impact compared with G-A in patients with tumor involving the greater curvature [32]. It is worth mentioning that the inclusion criteria of those studies were not rigorous in selecting patients. As a result, the conclusions of these studies should be explained with cautious. Thus, large well-designed studies are needed to explore the role of No.10 lymph node clearance in patients with possible splenic hilar lymph node metastasis in the future.

It is a matter of debate whether the spleen should be preserved or removed in prophylactic splenic hilar lymph node dissection. Supporters of splenectomy argued that $\mathrm{G}+\mathrm{S}$ could facilitate dissection of lymph nodes at the splenic hilum and along the splenic artery more radically, while others thought that G + SPSHD was quite enough for splenic hilar lymph node dissection. Moreover, splenectomy has been reported to be associated with increased morbidity and mortality rates due to the importance of the spleen as a part of the immune system $[16,41]$. The Korean RCTs [42] enrolling 207 patients showed slightly but not significantly better survival in $\mathrm{G}+\mathrm{S}$ group than

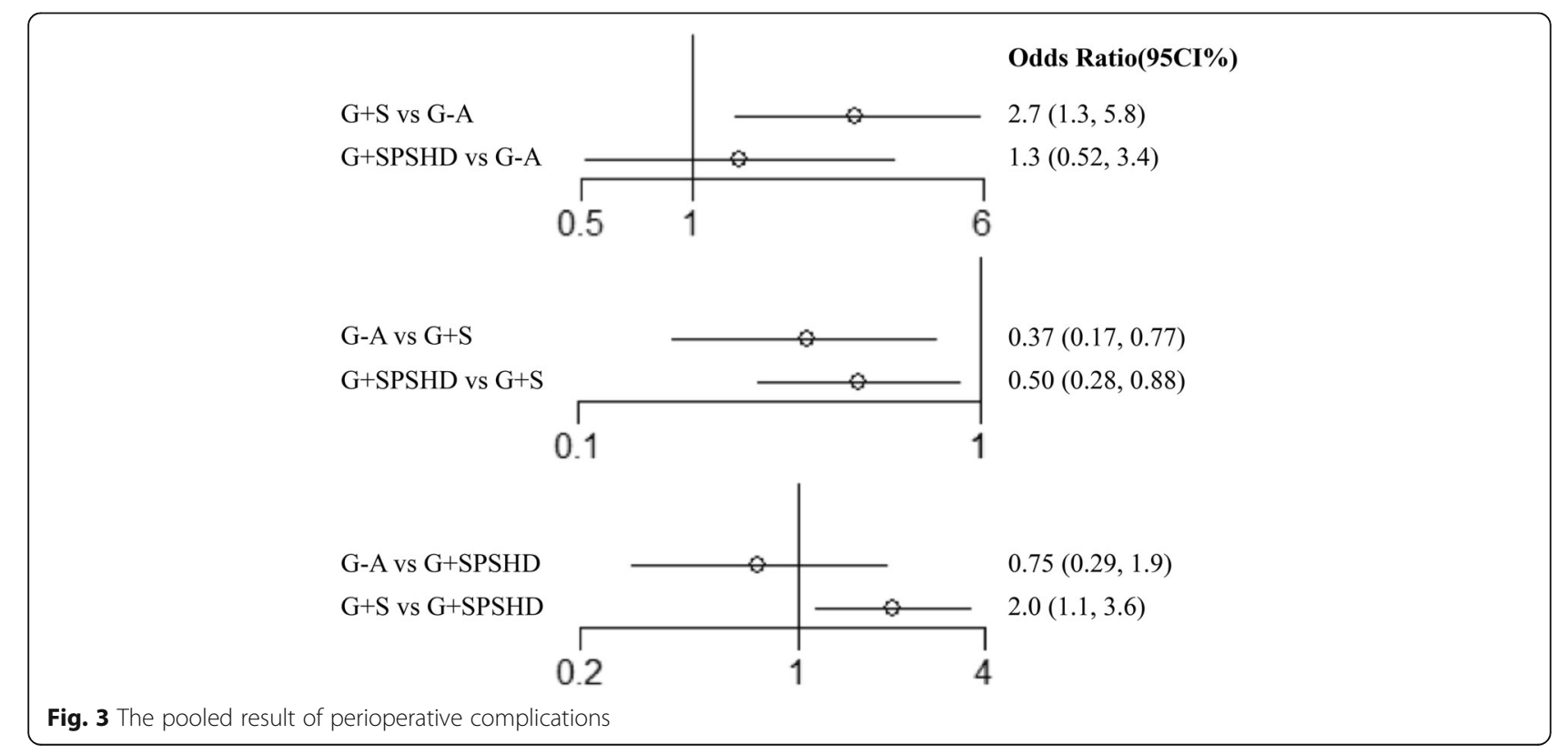




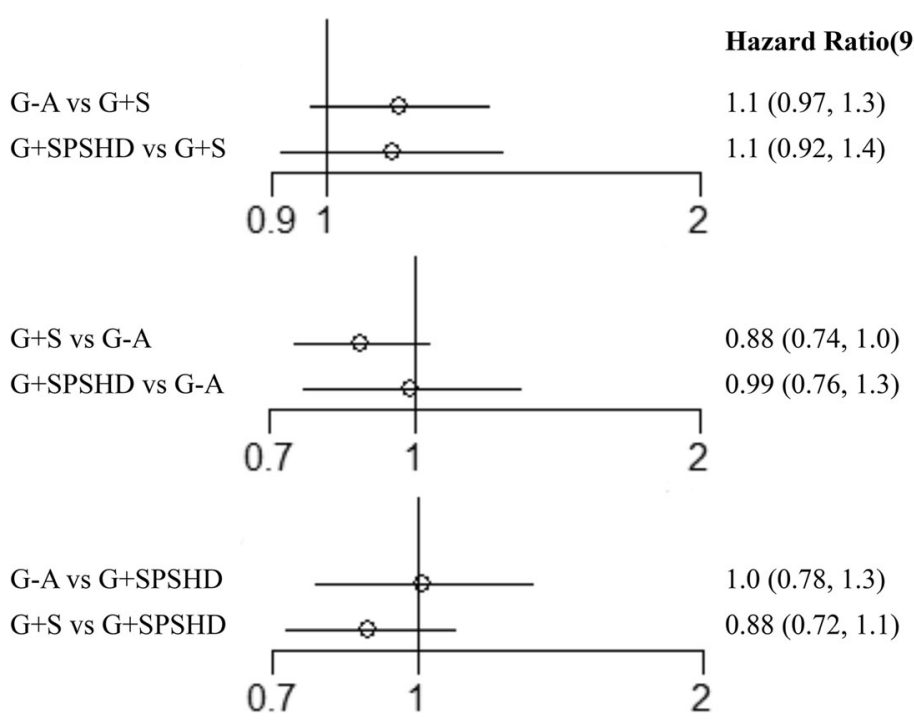

Fig. 4 The network meta-analyses of the 5-year overall survival rate

G + SPSHD group (54.8\% vs $48.8 \%$ ). Therefore, the study suggested that prophylactic lymphadenectomy with splenectomy was not justified, and spleen-preserved lymphadenectomy might be a better option for advanced upper and middle third gastric cancer patients. In our current study, $\mathrm{G}+\mathrm{S}$ also has no advantages in prophylactic splenic hilar lymph node dissection compared with G + SPSHD.

Study comparing the prognosis between G-A group and G + SPSHD group was limited. A retrospective study by Yang et al. reported that there was no significant difference of 5 -year survival rates between the two groups [43]. Another study by Bian et al. also found that G + SPSHD could not improve the overall survival compared with G-A in patients with advanced proximal gastric cancer without metastasis to No. $4 \mathrm{~s}$ lymph node. Meanwhile, G-A group had better short-term outcomes, faster recovery, and lower postoperative morbidity rates than G + SPSHD group [44]. However, the two studies involved a few patients with gross invasion of the splenic hilum, so we excluded them in our present research. In our indirect comparison metaanalyses, the total complication rate and 5-year survival rate of G + SPSHD group did not differ significant from that of G-A group. However, according to the results of cumulative ranking probability plots, the G-A has highest probability to be optimal surgical procedure for patients with gastric cancer. What's more, with respect to the safety, the fragile texture of the spleen and large amount of vessel branches being located at the splenic hilum may increase the risk of No. 10 lymphadenectomy.

There was only one meta-analysis which based on three RCTs evaluated the impact of splenectomy on long-term survival of patients with gastric cancer in the literature [45]. Yang et al. concluded that splenectomy did not show a beneficial effect on survival rates compared to splenic preservation. However, in their metaanalysis study, they failed to make a distinction between the G-A group and G + SPSHD group, and classified them as spleen-preserving group.

There are several limitations in our present study. First, the investigations enrolled in our network metaanalysis were all retrospective studies which introduces a possible limitation of selection bias, detection bias, and

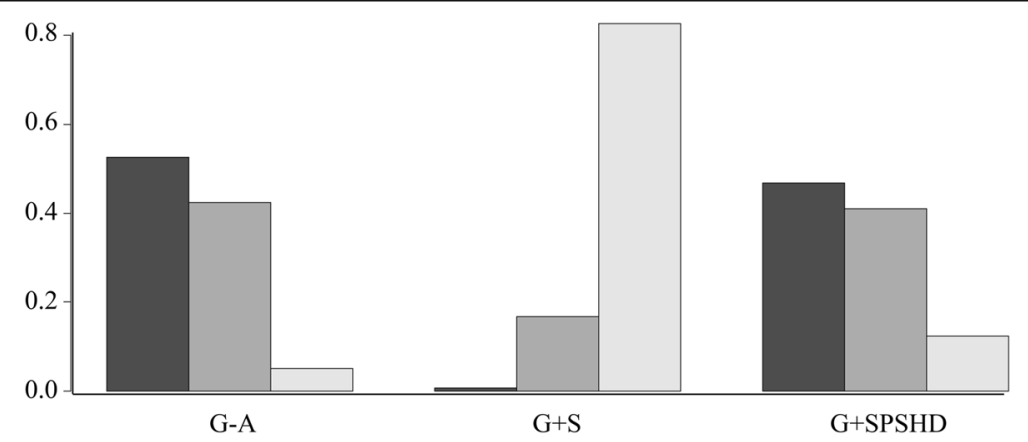

Fig. 5 Barplots for the ranking probabilities of each treatment 
performance of analysis bias. Second, we focused on overall survival only and did not analyze progressionfree survival. This was partly due to literature limitations, as some studies did not report progression-free survival for one or both groups. Third, according to our inclusion criteria, the tumors were not strictly limited to upper third of the stomach.

\section{Conclusion}

This network meta-analysis systemically reviewed currently available evidence for treatment of gastric cancer in the second-line setting to address the knowledge gap regarding the optimal extent of lymphadenectomy for these patients. Through both direct and indirect comparisons, we demonstrated that prophylactic $\mathrm{G}+\mathrm{S}$ and G + SPSHD were not recommended for treatment of middle and upper third gastric cancer.

\section{Additional file}

Additional file 1. Assessment of trace plots and the Brooks-GelmanRubin statistic.

\section{Abbreviations}

Cl: Confidence interval; G + S: Gastrectomy combination with splenectomy; G + SPSHD: Gastrectomy combination with spleen-preserving splenic hilar dissection; G-A: Gastrectomy alone; HR: Hazard ratio; LNM: Lymph node metastasis; NOS: Newcastle-Ottawa Scale; OR: Odds ratio; PRISMANMA: Preferred Reporting Items for Systematic Reviews and Meta-Analyses for Network Meta-Analyses; RCT: Randomized controlled trial; SHLNM: Splenic hilar lymph node metastasis; SMD: Standardized mean difference; SPSHD: Splenectomy or spleen-preserving splenic hilar dissection

\section{Acknowledgments}

None.

\section{Authors' contributions}

$Z G, L$ and $G Y$ drafted the manuscript, $Z G$ and $\amalg$ searched the literature, $Z G$, GY, WF and LS screened all titles and abstracts for eligibility, ZG and XG reviewed the main reports and supplementary materials, GM and LX performed statistical analysis. ZH and FF designed and supervised the study. All authors have read and approved the manuscript.

\section{Funding}

Not Applicable.

\section{Availability of data and materials}

Please contact the author Hongwei Zhang (zhenghwfmmu@126.com) upon reasonable requests.

\section{Ethics approval and consent to participate}

This study was approved by the Ethics Committee of Xijing Hospital.

\section{Consent for publication}

Not Applicable.

\section{Competing interests}

The authors declare that they have no competing interests.

\section{Author details}

${ }^{1}$ Department of Digestive Surgery, Xijing Hospital of Digestive Diseases, the Fourth Military Medical University, 127 West Changle Road, Xi'an 710032, Shaanxi, China. ${ }^{2}$ Cadre' s sanitarium, 62101 Army of PLA, 67 Nahu Road, Xinyang 464000, Henan, China. ${ }^{3}$ Health company, 92667 Army of PLA, 39
East Zaoshan Road, Qingdao 266100, Shandong, China. ${ }^{4}$ Department of General Surgery, No. 534 Hospital of PLA, West Lichun Road, Luoyang 471000, Henan, China.

Received: 5 October 2019 Accepted: 11 February 2020

Published online: 24 February 2020

\section{References}

1. Jemal A, Bray F, Center MM, et al. Global cancer statistics. CA Cancer J Clin. 2011;61:69-90.

2. Wu CW, Hsiung CA, Lo SS, et al. Nodal dissection for patients with gastric cancer: a randomised controlled trial. Lancet Oncol. 2006;7:309-15.

3. Huang CM, Lin JX, Zheng $\mathrm{CH}$, et al. Effect of negative lymph node count on survival for gastric cancer after curative distal gastrectomy. Eur J Surg Oncol. 2011:37:481-7.

4. Monig SP, Collet PH, Baldus SE, et al. Splenectomy in proximal gastric cancer: frequency of lymph node metastasis to the splenic hilus. J Surg Oncol. 2001;76:89-92.

5. Ikeguchi M, Kaibara N. Lymph node metastasis at the splenic hilum in proximal gastric cancer. Am Surg. 2004;70:645-8.

6. Shin $\mathrm{SH}$, Jung $\mathrm{H}$, Choi $\mathrm{SH}$, et al. Clinical significance of splenic hilar lymph node metastasis in proximal gastric cancer. Ann Surg Oncol. 2009;16:1304-9.

7. Watanabe M, Kinoshita T, Enomoto N, et al. Clinical significance of splenic hilar dissection with splenectomy in advanced proximal gastric cancer: an analysis at a single institution in Japan. World J Surg. 2016;40:1165-71.

8. Sasada S, Ninomiya M, Nishizaki M, et al. Frequency of lymph node metastasis to the splenic hilus and effect of splenectomy in proximal gastric cancer. Anticancer Res. 2009:29:3347-51.

9. Ohno M, Nakamura T, Ajiki T, et al. Procedure for lymph node dissection around splenic artery in proximal gastric cancer. Hepatogastroenterology. 2003;50:1173-7.

10. Noguchi Y, Imada T, Matsumoto A, et al. Radical surgery for gastric cancer. A review of the Japanese experience. Cancer. 1989:64:2053-62.

11. Soga J, Kobayashi K, Saito J, et al. The role of lymphadenectomy in curative surgery for gastric cancer. World J Surg. 1979:3:701-8.

12. Roukos DH. Current advances and changes in treatment strategy may improve survival and quality of life in patients with potentially curable gastric cancer. Ann Surg Oncol. 1999;6:46-56.

13. Toge $T$, Hamamoto S, Itagaki $E$, et al. Analysis of suppressor cell activities in spleen cells from gastric cancer patients and the effect of splenectomy on prognosis of gastric cancer. Nihon Geka Gakkai Zasshi. 1983;84:961-4.

14. Lee KY, Noh SH, Hyung WJ, et al. Impact of splenectomy for lymph node dissection on long-term surgical outcome in gastric cancer. Ann Surg Oncol. 2001:8:402-6.

15. Brady MS, Rogatko A, Dent LL, Shiu MH. Effect of splenectomy on morbidity and survival following curative gastrectomy for carcinoma. Arch Surg. 1991; 126:359-64

16. Otsuji E, Yamaguchi T, Sawai $\mathrm{K}$, et al. End results of simultaneous splenectomy in patients undergoing total gastrectomy for gastric carcinoma. Surgery. 1996;120:40-4.

17. Stang A. Critical evaluation of the Newcastle-Ottawa scale for the assessment of the quality of nonrandomized studies in meta-analyses. Eur J Epidemiol. 2010;25:603-5.

18. Hutton B, Salanti G, Caldwell DM, et al. The PRISMA extension statement for reporting of systematic reviews incorporating network meta-analyses of health care interventions: checklist and explanations. Ann Intern Med. 2015; 162:777-84.

19. Neupane B, Richer D, Bonner AJ, et al. Network meta-analysis using R: a review of currently available automated packages. PLoS One. 2014;9:e115065.

20. Cooper NJ, Sutton AJ, Lu G, Khunti K. Mixed comparison of stroke prevention treatments in individuals with nonrheumatic atrial fibrillation. Arch Intern Med. 2006;166:1269-75.

21. Higgins JP, Whitehead A. Borrowing strength from external trials in a metaanalysis. Stat Med. 1996;15:2733-49.

22. Lu G, Ades AE. Combination of direct and indirect evidence in mixed treatment comparisons. Stat Med. 2004:23:3105-24.

23. Gelman SPBaA. General methods for monitoring convergence of iterative simulations; 2012

24. Parmar MK, Torri V, Stewart L. Extracting summary statistics to perform meta-analyses of the published literature for survival endpoints. Stat Med. 1998;17:2815-34. 
25. Chaimani A, Higgins JP, Mavridis D, et al. Graphical tools for network metaanalysis in STATA. PLoS One. 2013;8:e76654.

26. Higgins JP, Thompson SG, Deeks JJ, Altman DG. Measuring inconsistency in meta-analyses. Bmj. 2003;327:557-60.

27. Erturk S, Ersan Y, Cicek Y, et al. Effect of simultaneous splenectomy on the survival of patients undergoing curative gastrectomy for proximal gastric carcinoma. Surg Today. 2003;33:254-8.

28. Ji F, Zhang J, Wen D, et al. Effect of splenectomy on the survival of patients underwent radical surgery for gastric cardia cancer. Int J Clin Exp Med. 2016; 9:22217-21.

29. Kodera Y, Yamamura Y, Shimizu Y, et al. Lack of benefit of combined pancreaticosplenectomy in D2 resection for proximal-third gastric carcinoma. World J Surg. 1997;21:622-7 discussion 627-628.

30. Kwon SJ. Prognostic impact of splenectomy on gastric cancer: results of the Korean Gastric Cancer Study Group. World J Surg. 1997;21:837-44.

31. Oh SJ, Hyung WJ, Li C, et al. The effect of spleen-preserving lymphadenectomy on surgical outcomes of locally advanced proximal gastric cancer. J Surg Oncol. 2009;99:275-80.

32. Ohkura Y, Haruta S, Shindoh J, et al. Efficacy of prophylactic splenectomy for proximal advanced gastric cancer invading greater curvature. World I Surg Oncol. 2017;15:106.

33. Yao XX, Sah BK, Yan M, et al. Radical gastrectomy with combined splenectomy: unnecessary. Hepatogastroenterology. 2011;58:1067-70.

34. Son SY, Shin DJ, Park YS, et al. Spleen-preserving lymphadenectomy versus splenectomy in laparoscopic total gastrectomy for advanced gastric cancer. Surg Oncol. 2017;26:207-11.

35. Fang WL, Huang KH, Wu CW, et al. Combined splenectomy does not improve survival in radical total gastrectomy for advanced gastric cardia cancer. Hepatogastroenterology. 2012;59:1150-4.

36. Csendes A, Burdiles P, Rojas J, et al. A prospective randomized study comparing D2 total gastrectomy versus D2 total gastrectomy plus splenectomy in 187 patients with gastric carcinoma. Surgery. 2002;131:401-7.

37. Sano T, Sasako M, Mizusawa J, et al. Randomized controlled trial to evaluate splenectomy in total gastrectomy for proximal gastric carcinoma. Ann Surg. 2017:265:277-83

38. Toge T, Kameda A, Kuroi K, et al. The role of the spleen in immunosuppression and the effects of splenectomy on prognosis in gastric cancer patients. Nihon Geka Gakkai Zasshi. 1985;86:1120-3.

39. Ito $\mathrm{H}$, Inoue $\mathrm{H}$, Odaka $\mathrm{N}$, et al. Prognostic impact of prophylactic splenectomy for upper-third gastric cancer: a cohort study. Anticancer Res. 2013;33:277-82

40. Nashimoto A, Yabusaki H, Matsuki A. The significance of splenectomy for advanced proximal gastric cancer. Int J Surg Oncol. 2012;2012:301530.

41. Ellison EC, Fabri PJ. Complications of splenectomy. Etiology, prevention, and management. Surg Clin North Am. 1983;63:1313-30.

42. Yu W, Choi GS, Chung HY. Randomized clinical trial of splenectomy versus splenic preservation in patients with proximal gastric cancer. Br J Surg. 2006; 93:559-63.

43. Yang $\mathrm{K}$, Zhang $\mathrm{WH}$, Chen $\mathrm{XZ}$, et al. Survival benefit and safety of no. 10 lymphadenectomy for gastric cancer patients with total gastrectomy. Medicine (Baltimore). 2014;93:e158.

44. Bian $\mathrm{S}, \mathrm{Xi} H, \mathrm{Wu} X$, et al. The role of no. 10 lymphadenectomy for advanced proximal gastric cancer patients without metastasis to no. 4sa and no. 4sb lymph nodes. J Gastrointest Surg. 2016;20:1295-304.

45. Yang K, Chen XZ, Hu JK, et al. Effectiveness and safety of splenectomy for gastric carcinoma: a meta-analysis. World J Gastroenterol. 2009;15:5352-9.

\section{Publisher's Note}

Springer Nature remains neutral with regard to jurisdictional claims in published maps and institutional affiliations.

Ready to submit your research? Choose BMC and benefit from:

- fast, convenient online submission

- thorough peer review by experienced researchers in your field

- rapid publication on acceptance

- support for research data, including large and complex data types

- gold Open Access which fosters wider collaboration and increased citations

- maximum visibility for your research: over $100 \mathrm{M}$ website views per year

At BMC, research is always in progress.

Learn more biomedcentral.com/submissions 